\title{
Population Protocols with Faulty Interactions: the Impact of a Leader*
}

\author{
Giuseppe Antonio Di Luna ${ }^{\dagger} \quad$ Paola Flocchini ${ }^{\dagger} \quad$ Taisuke Izumi $^{\ddagger}$ \\ Tomoko Izumi $^{\S}$ Nicola Santoro Giovanni Viglietta ${ }^{\dagger}$
}

\begin{abstract}
We consider the problem of simulating traditional population protocols under weaker models of communication, which include one-way interactions (as opposed to two-way interactions) and omission faults (i.e., failure by an agent to read its partner's state during an interaction), which in turn may be detectable or undetectable. We focus on the impact of a leader, and we give a complete characterization of the models in which the presence of a unique leader in the system allows the construction of simulators: when simulations are possible, we give explicit protocols; when they are not, we give proofs of impossibility. Specifically, if each agent has only a finite amount of memory, the simulation is possible only if there are no omission faults. If agents have an unbounded amount of memory, the simulation is possible as long as omissions are detectable. If an upper bound on the number of omissions involving the leader is known, the simulation is always possible, except in the one-way model in which one side is unable to detect the interaction.
\end{abstract}

\section{Introduction}

\subsection{Framework}

Consider a system of interacting computational entities, called agents, whose interaction is however not under their control but decided by an external scheduler. Such are for example systems of wireless mobile entities where two entities can interact (i.e., exchange information) when their movement brings them into communication range of each other, but their movements, and thus their interactions, are unpredictable. Systems satisfying this condition, sometimes called opportunistic mobility or passive mobility, have been extensively examined under variety of assumptions, especially within the context of distributed computing in highly dynamic networks and time-varying graphs (for recent surveys see [13, 19]).

In particular, in the population protocol model (PP), introduced in the seminal paper [3], the entities are assumed to be finite-state and anonymous (i.e., identical), execute the same protocol, and interactions are always between pairs of agents. The roles of the two agents involved in an interaction are asymmetric: one agent is considered the starter and the other is the reactor. Still, the communication is two-way: each agent receives the state of the other and executes the protocol to update its own state based on the received information and its own state. Furthermore, in the selection of the occurrences of the interactions, the scheduler is constrained to satisfy some fairness assumption.

The restricted computational universe defined by the basic assumptions of PP has been subsequently expanded in an attempt to overcome the inherent computability limitations and to examine the computational impact of factors such as non-constant memory (e.g., [1, 2, 15), presence of a leader (e.g., 7), storage of information on edges (e.g., [16, 17, 18), etc.

In all these models, including the original one, the interaction is assumed to be fault-free. An immediate important question is what happens if interactions are subject to failures.

Very little is know in this regard. An insight comes from the study of the so-called one-way interaction models [4, where the starter of an interaction is not able to see the state of the reactor (immediate transmission), or it is not even able to detect that the interaction has taken place (immediate observation). This study showed that, under

\footnotetext{
* This work was supported in part by NSERC Discovery Grants, and by KAKENHI No. 15H00852 and 25289227.

$\dagger$ University of Ottawa. E-mails: \{gdiluna, paola.flocchini, gvigliet\}@uottawa.ca.

${ }^{\ddagger}$ Nagoya Institute of Technology, Gokiso-cho, Showa-ku, Nagoya, Aichi, 466-8555, Japan. E-mail: t-izumi@nitech.ac.jp.

$\S$ Ritsumeikan University. E-mail: izumi-t@fc.ritsumei.ac.jp.

๑Carleton University. E-mail: santoro@scs.carleton.ca.
} 
one-way interactions, the computational power of the agents is strictly weaker than with the usual bidirectional interactions. In particular, if the interactions are not detectable by the starter (i.e., immediate observation), the agents can compute only the threshold predicates [4.

The one-way interaction models capture a class of permanent omission failures, those occurring at the starter's side. Clearly, there are many more types of omission failures, such as those occurring at the reactor's side and, more insidious, those whose occurrence is dynamic and unpredictable. And of course, for each of these types there are different variations, depending on the kind of fault detection assumed.

The complete range of dynamic omission failures has been classified in 24], where the following general question was posed: under what additional system capabilities is it possible to correctly execute every traditional two-way population protocol in spite of dynamic omission failures? More specifically, under what conditions (if any) it is possible to simulate the execution of every two-way population protocol for a given class of omission failures? The simulator should be a population protocol that, in each execution in the model defined by the considered class of omissions, produces a correct execution of any traditional two-way population protocol $\mathcal{P}$ given as input, regardless of the nature of $\mathcal{P}$ and the constraints its execution might have; and it does so unobtrusively at each agent, interacting with $\mathcal{P}$ only by observing its internal state, and providing to it the internal state of another agent. In other words, a simulator provides an interface between the simulated protocol $\mathcal{P}$ and the physical communication layer, giving the system the illusion of being in a fault-free two-way environment.

The existence of simulators is important in scenarios in which we do not only concern ourselves with the final output of a population protocol, but also with the execution that leads to the result. We may want, for instance, to guarantee that our simulating agents enter some critical states exactly as many times as they would if they were actually executing the protocol that is being simulated.

The existence of fault-tolerant one-way simulators of two-way protocols has been investigated in 24 in terms of the amount memory required by the agents to perform such simulations, and a variety of models and results were established. It is shown that, with no a-priori knowledge, the simulation of two-way protocols in the presence of omissions is impossible even if the agents have infinite memory. In the weakest models investigated, this impossibility holds even if the number of omission failures in each execution is limited to one. On the other hand, it is also shown that simulation is possible if agents have unique IDs or the total number of agents is known. Moreover, in some restricted models, simulation is possible when an upper bound on the number of omission faults is known.

In this paper we continue this general line of research and investigate how the presence in the system of a distinguished agent, a leader, can impact the capability of the system to tolerate dynamic omission failures. More precisely, we study the possibility and impossibility of simulation of two-way protocols with the aid of a leader, with respect to the different classes of omission failures and one-way interactions.

\subsection{Main Contributions}

As in 24, we consider all the computationally distinct models that arise from the introduction of omission faults and/or one-way interactions in two-way protocols: TW, IT, IO, $\mathbf{T}_{i}(i=1,2,3$,$) , and \mathbf{I}_{j}(j=1,2,3,4$,$) ; see Figure 1$. where the transition function $\delta$, detailed in Section 2, uniquely identifies each model. In particular, TW refers to two-way protocols without omissions; IT and IO refer to the one-way models immediate transmission and immediate observation, introduced in [4]; the $\mathrm{T}_{i}$ 's and $\mathrm{I}_{i}$ 's refer to the distinct two-way and one-way models with omissions, respectively.

We consider two types of omission adversaries: informally, a "malignant" one (UO), which is able to arbitrarily insert omission faults into "globally fair" sequences of interactions, and a "benign" one $(\diamond \mathbf{N O})$, which inserts some omission faults, but eventually stops. To make our results stronger, we always assume the benign adversary in in the impossibility proofs and the malignant one in the possibility proofs.

We study the negative impact that omissions have on computability, and we show that the simulation of two-way protocols is impossible even with the aid of a leader (Theorem 1), assuming that the amount of memory is bounded.

On the other hand, we show that the presence of both a leader and infinite memory on each agent makes the simulation possible in the weak intermediate one-way models $\mathrm{I}_{1}$ and $\mathrm{I}_{2}$ (Theorem 4), and thus in all the upper models of Figure 1. The fact that this possibility does not apply to $I O$ and $T_{1}$ is not accidental: indeed we prove that, for these two models, the simulation is impossible even with both a leader and infinite memory, even against the benign omission adversary (Theorem 2).

Finally, we study what happens when a bound on the omission failures involving the leader is known, and essentially we show that simulators exists for models $I_{1}$ and $I_{2}$ (Theorem 5 ) and model $T_{1}$ (Theorem 6), and these 
imply the possibility of simulations in all other omissive models.

For non-omissive models, we show that two-way simulation is possible in the IT model (Theorem 7). In light of the fact that with constant memory, in absence of additional capabilities, IT protocols are strictly less powerful than TW (see [24]), our results show that this computational gap can be overcome by using a leader.

Our main results are summarized in Figure 2, where white blobs represent possibilities, and gray blobs impossibilities. As a consequence of these results, we have a complete characterization of the feasibility of simulations with respect to the presence of a leader.

\section{$1.3 \quad$ Related Work}

Starting with the seminal paper [8, there have been extensive investigations on population protocols (e.g., see [5, 11, 14, 19, 20, 21, 26]). In order to overcome the inherent computability restrictions of the model, several extensions have been proposed. For example, endowing each agent with non-constant memory [1, 2, 15, assuming the presence of a leader [7, allowing a certain amount of information to be stored on the edges of the "communication graph" [16, 17, 18, etc.

The possibility of reliable computations in PP, first considered in [22, has been studied only with respect to processors' faults, and the basic model has necessarily been expanded. In 23] it has been shown how to compute functions tolerating $\mathcal{O}(1)$ crash stops and transient failures, assuming that the number of failures is bounded and known. In [6] the majority problem under $\mathcal{O}(\sqrt{n})$ Byzantine failures, assuming a fair probabilistic scheduler, has been studied. In [27] unique IDs are assumed, and it is shown how to compute functions tolerating a bounded number of Byzantine faults, under the assumption that Byzantine agents cannot forge IDs. Self-stabilizing solutions have been devised for specific problems such as: leader election, assuming knowledge of the system size and a nonconstant number of states [12, or assuming a leader detection oracle 25]; counting, assuming the presence of a leader [9. Moreover, in [10] a self-stabilizing transformer for general protocols has been studied in a slightly different model and under the assumption of unbounded memory and a leader.

Finally, to the best of our knowledge, the one-way model, without omissions, has been studied only in [4, where it is shown that IT and IO, when equipped with constant memory, can compute a set of functions that is strictly included in that of TW. The omission models that we consider have been introduced for the first time in 24, where a characterisation of what can be simulated without a leader is given. Our paper complements and enriches the results of [24], showing what additional power is obtained assuming the presence of a leader.

\section{Model and Terminology}

In this section we briefly define the computation model, the notion of omission, and the notion of simulator. Due to space constraints, we do not include all the formal definitions, which can be found in 24 .

\subsection{Interacting Entities}

We consider a system consisting of a set $A=\left\{a_{1}, \ldots, a_{n}\right\}$ of interacting computational entities, called agents. Each interaction involves only two agents with asymmetric roles: one agent is the starter and the other is the reactor. Interactions occur at discrete times, and at every "time unit" exactly one interaction occurs. The starter and the reactor of each interaction are chosen by an external "adversarial scheduler" in a "globally fair" way (see 24] for details).

When two agents interact, they exchange information and perform a local computation according to the same protocol $\mathcal{P}$. A protocol is a pair $\mathcal{P}=\left(Q_{\mathcal{P}}, \delta_{\mathcal{P}}\right)$, where $Q_{\mathcal{P}}$ is a set of local states and $\delta_{\mathcal{P}}: Q_{\mathcal{P}} \times Q_{\mathcal{P}} \rightarrow Q_{\mathcal{P}} \times Q_{\mathcal{P}}$ is the transition function defining the states of the two interacting agents at the end of their local computation. Some elements of $Q_{\mathcal{P}}$ are labeled as "initial states"; when the execution of the protocol begins, all agents have (any combination of) initial states. With a small abuse of notation, and when no ambiguity arises, we will use the same literal (e.g., $a_{i}$ ) to indicate both an agent and its local state. A configuration of $\mathcal{P}$ is a multiset of local states of $\mathcal{P}$.

We can model the presence of a leader in the system by stipulating that, in every initial configuration, there is exactly one agent in a distinguished state (or set of states).

Depending on the conditions imposed on the transition function, three main models of interactions have been identified: the standard two-way model and the one-way models, immediate transmission and immediate observation, presented in [4]. 
Two-Way Interaction Model (TW). The state transition function consists of two functions $f_{s}: Q_{\mathcal{P}} \times Q_{\mathcal{P}} \rightarrow$ $Q_{\mathcal{P}}$ and $f_{r}: Q_{\mathcal{P}} \times Q_{\mathcal{P}} \rightarrow Q_{\mathcal{P}}$, one for the starter and the other for the receiver respectively, with $\delta_{\mathcal{P}}\left(a_{s}, a_{r}\right)=$ $\left(f_{s}\left(a_{s}, a_{r}\right), f_{r}\left(a_{s}, a_{r}\right)\right)$.

Immediate Transmission Model (IT). The state transition function consists of two functions $g: Q_{\mathcal{P}} \rightarrow Q_{\mathcal{P}}$ and $f: Q_{\mathcal{P}} \times Q_{\mathcal{P}} \rightarrow Q_{\mathcal{P}}$, with $\delta_{\mathcal{P}}\left(a_{s}, a_{r}\right)=\left(g\left(a_{s}\right), f\left(a_{s}, a_{r}\right)\right)$. Note that, in the IT interaction model, the starter does not read the state of the reactor but it explicitly detects the interaction, as it applies function $g$ to its own state.

Immediate Observation Model (IO). The state transition function has the form $\delta_{\mathcal{P}}\left(a_{s}, a_{r}\right)=\left(a_{s}, f\left(a_{s}, a_{r}\right)\right)$. Note that, in the 10 model, there is no detection of the interaction (or proximity) by the starter.

\subsection{Omissions}

An omission is a fault involving a single interaction. In an omissive interaction, an agent does not receive any information about the state of the other. If omissions can occur in the system, then transition functions become more general relations.

Two-Way Omissive Models. In the most general omissive model, $\mathrm{T}_{3}$, the transition relation has the form

$$
\delta\left(a_{s}, a_{r}\right)=\left\{\left(f_{s}\left(a_{s}, a_{r}\right), f_{r}\left(a_{s}, a_{r}\right)\right),\left(o\left(a_{s}\right), f_{r}\left(a_{s}, a_{r}\right)\right),\left(f_{s}\left(a_{s}, a_{r}\right), h\left(a_{r}\right)\right),\left(o\left(a_{s}\right), h\left(a_{r}\right)\right)\right\} .
$$

The first pair is the outcome of an interaction when no omission is present; the other three pairs represent all possible outcomes when there is an omission: respectively, an omission on the starter's side, on the reactor's side, and on both sides. The functions $o$ and $h$ represent the detection capabilities of each agent: if one of these is the identity, then omissions are undetectable on the respective side. This gives rise to the weaker models $\mathrm{T}_{2}$ and $\mathrm{T}_{1}$ depicted in Figure 1 (see 24] for more details).

One-Way Omissive Models. These models are defined by the transition relation

$$
\delta\left(a_{s}, a_{r}\right)=\left\{\left(g\left(a_{s}\right), f\left(a_{s}, a_{r}\right)\right),\left(o\left(a_{s}\right), h\left(a_{r}\right)\right)\right\} .
$$

The first pair is the outcome of an interaction when no omission is present, and the second pair when there is an omission. Note that the $\mathrm{IO}$ model corresponds to the case in which $g$ is the identity function and there are no omissions. Once again, omissions are undetectable starter-side if $o$ is the identity function or if $o=g$. Moreover, if $h=g$, the reactor has detected the proximity of another agent, but is unable to read its state or even determine who is the starter and who is the reactor. Collectively, these variations give rise to models $I_{1}$ to $I_{4}$ in Figure 1 . Other combinations of omissions and detections are possible, but they are provably equivalent to some of the aforementioned ones (see 24] for more details).

Omissions are introduced by an adversarial entity. We consider two types of adversaries:

(1) the Unfair Omissive Adversary (UO), which arbitrarily inserts omissive interactions in any execution, and

(2) the Eventually Non-Omissive Adversary $(\diamond \mathbf{N O})$, which can only insert finitely many omissions in an execution.

\subsection{Simulation of Two-Way Protocols}

Let $\mathcal{P}$ be a two-way protocol, and let $\mathcal{S}(\mathcal{P})$ be any protocol (which could be one-way, omissive, or both). Next we are going to informally define what it means for $\mathcal{S}(\mathcal{P})$ to simulate $\mathcal{P}$ (for a formal definition, refer to [24]).

We want the set of local states of $\mathcal{S}(\mathcal{P})$ to be of the form $Q_{\mathcal{P}} \times Q_{\mathcal{S}}$, where $Q_{\mathcal{P}}$ is the set of local states of $\mathcal{P}$ (the "simulated states"), and $Q_{\mathcal{S}}$ is some additional memory space used in the simulation. Suppose now to start an execution of $\mathcal{S}(\mathcal{P})$ on a system of $n>2$ agents from a given initial configuration. Agents are allowed to freely change the $Q_{\mathcal{S}}$ component of their local states; but when they change their $Q_{\mathcal{P}}$ component, we want the change to reflect the transition function of $\mathcal{P}$. That is, if $\delta_{\mathcal{P}}\left(a_{s}, a_{r}\right)=\left(f_{s}\left(a_{s}, a_{r}\right), f_{r}\left(a_{s}, a_{r}\right)\right)$, then for every agent whose simulated state changes from $a_{s}$ to $f_{s}\left(a_{s}, a_{r}\right)$, there must be some other agent (at some point in time) whose simulated state changes from $a_{r}$ to $f_{r}\left(a_{s}, a_{r}\right)$. Moreover, there must be a perfect matching between such transitions, in such a way that each starter of a simulated transition can be implicitly mapped to an appropriate reactor. Also, such a perfect matching must be "temporally consistent", i.e., there must be an ordering of the simulated two-way interactions that respects the order of the local state changes of each agent.

We additionally require that, if the execution of $\mathcal{S}(\mathcal{P})$ is globally fair (in the sense defined in [24]), then also the resulting simulated execution of $\mathcal{P}$ is globally fair. 


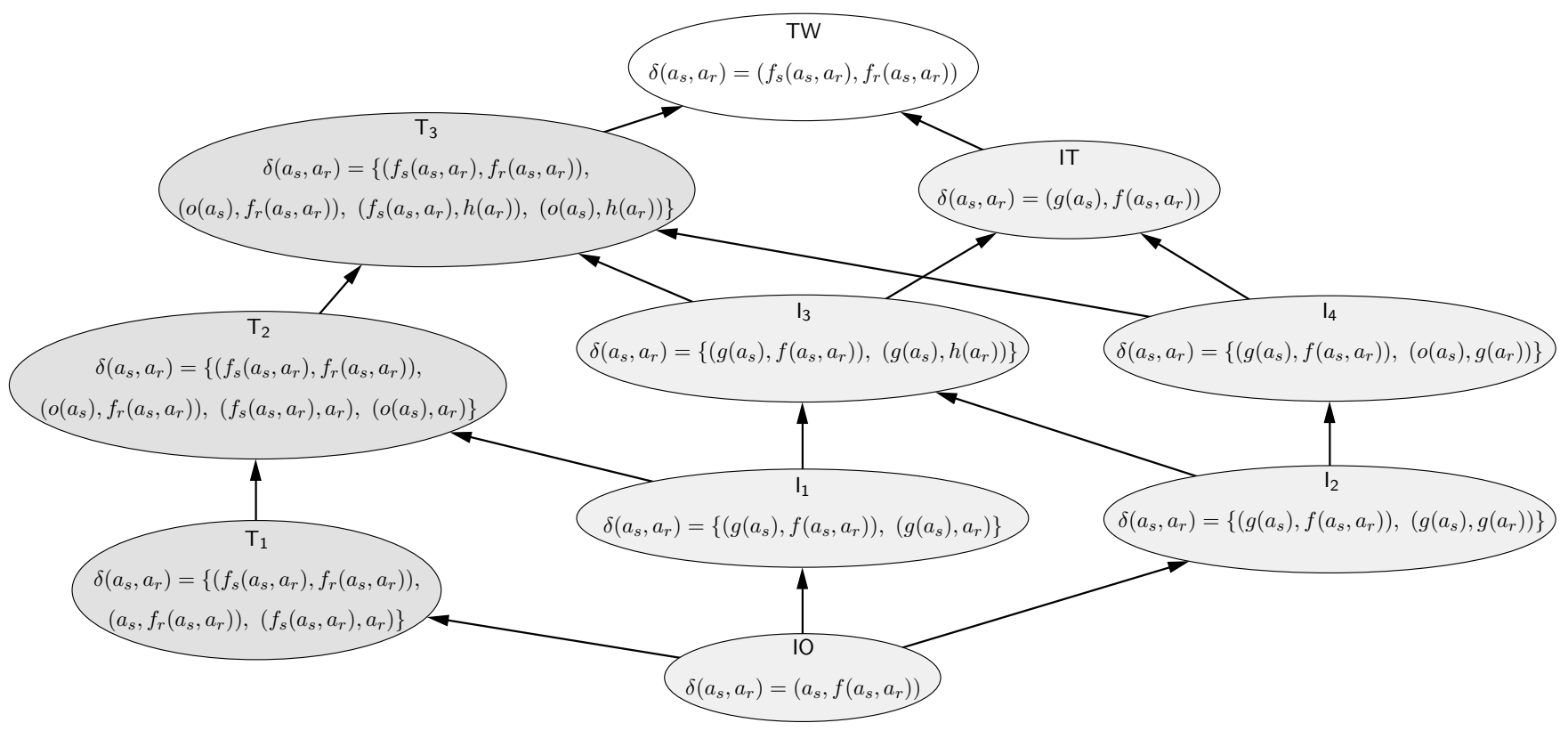

Figure 1: Interaction models (up to equivalence) and their computational relationships. An arrow between two blobs indicates that the class of solvable problems in the source blob is included in that of the destination blob. The models on the left, $T_{1}, T_{2}, T_{3}$, are the two-way models with omissions. The models on the right, $I_{1}, I_{2}, I_{3}, I_{4}$, are the one-way models with omissions.

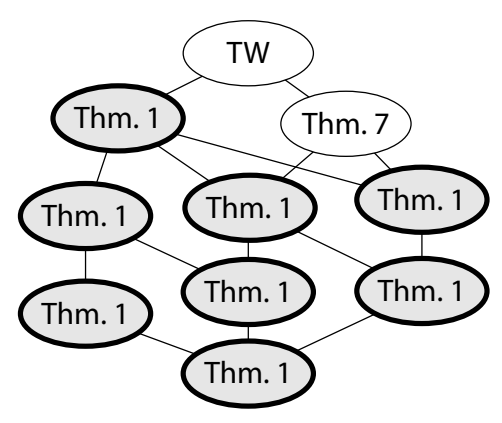

Finite memory

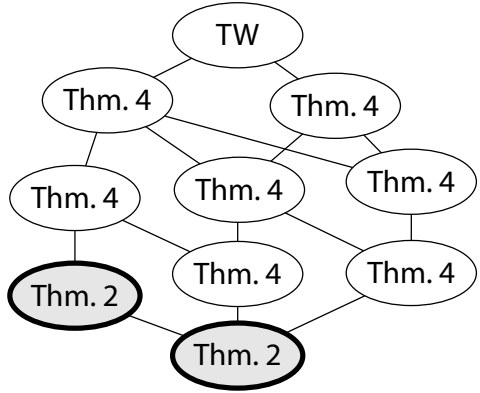

Infinite memory

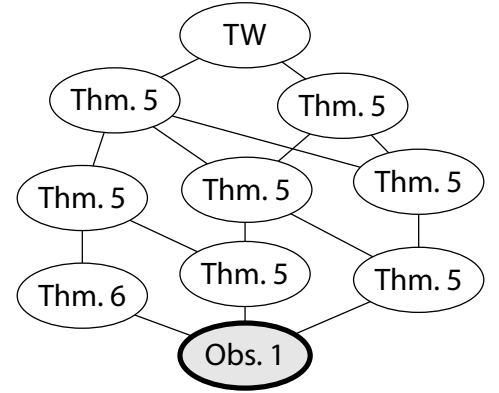

Knowledge on omissions

Figure 2: Map of results (cf. Figure 11). White blobs denote the existence of simulators; gray blobs indicate that simulations are not possible.

\section{Simulation with a Leader in Omissive Models: Impossibility}

In this section we prove that the presence of a leader, alone, might not be sufficient to overcome dynamic omissions. Indeed, we prove that there are two-way protocols that cannot be simulated with omissive interactions even if a leader is present.

Next we consider the Pairing Problem introduced in [24]: a set of agents $A$ is given, partitioned into consumer agents $A_{c}$, starting in state $c$, and producer agents $A_{p}$, starting in state $p$. We say that a protocol $\mathcal{P}$ solves the Pairing Problem if it enforces the following properties:

(i) Irrevocability: $\mathcal{P}$ has a state $c s$ that only agents in state $c$ can reach; once an agent has state $c s$, its state cannot change any more; 
(ii) Safety: At any time, the number of agents in state $c s$ is at most $\left|A_{p}\right|$;

(iii) Liveness: In all globally fair executions of $\mathcal{P}$, eventually the number of agents in state $c s$ is stably equal to $\min \left\{\left|A_{c}\right|,\left|A_{p}\right|\right\}$.

This problem can be solved in the standard two-way model by the simple protocol below:

Pairing Protocol $\mathcal{P}_{I P} . Q_{\mathcal{P}_{I P}}=\{c s, c, p, \perp\}$. The only non-trivial transition rules are $(c, p) \mapsto(c s, \perp)$ and $(p, c) \mapsto(\perp, c s)$.

However, as we will show, this protocol cannot be simulated, in spite of the presence of a leader, even in the simplest of the omissive models.

\subsection{Impossibility with Finite Memory}

We investigate what happens when we introduce a distinguished leader node, but we restrict the memory of agents to be bounded by some function of $|A|$. We show our impossibility results directly for the $\mathrm{T}_{3}$ omissive model. The results clearly carry over to all the less powerful omissive models.

Definition 1. (Omission-Recurrent Configuration) Let $\{\ell, a\}$ be a system of two agents, where $\ell$ is the leader, and let $C=\left(q_{\ell}, q_{a}\right)$ be a configuration. Suppose that there exists a finite non-empty sequence of interactions $I=\left(i_{1}, i_{2}, \ldots, i_{t}\right)$, where $i_{1}$ is omissive on both sides, such that, if $I$ is executed according to the transition rules of $\mathcal{P}$ starting from configuration $C$, eventually the state of $\ell$ is again $q_{\ell}$. Then, if $\ell$ is the starter (respectively, the reactor) of $i_{1}$, we say that $C$ is a starter-omission-recurrent configuration (respectively, an reactor-omissionrecurrent configuration) for protocol $\mathcal{P}$.

Note that, in the above definition, since $i_{1}$ is omissive on both sides, the system transitions into configuration $\left(o\left(q_{\ell}\right), h\left(q_{a}\right)\right)$ or $\left(h\left(q_{\ell}\right), o\left(q_{a}\right)\right)$ after executing $i_{1}$.

Lemma 1. Let $\mathcal{S}(\mathcal{P})$ be a simulator having a finite number $k$ of states in total and working under the $\diamond \mathbf{N O}$ adversary. Let a system of two agents $\{\ell, a\}$ be given, where $\ell$ is the leader. Let $C_{0}$ be an initial configuration for $\ell$ and $a$, and let $I=\left(i_{1}, i_{2}, \ldots\right)$ be an infinite sequence of interactions with no omissions between $\ell$ and a such that, if $\mathcal{S}(\mathcal{P})$ is executed according to I starting from $C_{0}$, then the execution is globally fair. Then there exists a finite sequence of interactions $I^{\prime}=\left(i_{1}^{\prime}, i_{2}^{\prime}, \ldots i_{t}^{\prime}\right)$ with the following properties.

(1) $I^{\prime}$ is obtained by introducing at most $k$ omissive interactions into an initial finite sub-sequence of $I$. All the omissive interactions of $I^{\prime}$ are omissive on both sides.

(2) If $\ell$ and a execute $\mathcal{S}(\mathcal{P})$ according to $I^{\prime}$ starting from $C_{0}$, they both do a simulated state transition (according to $\left.\delta_{\mathcal{P}}\right)$.

(3) Suppose that $\ell$ and a execute $\mathcal{S}(\mathcal{P})$ according to $I^{\prime}$ starting from $C_{0}$, and let $C_{j}$ be the configuration of $\ell$ and a immediately before executing interaction $i_{j+1}^{\prime}$. Then, if $i_{j+1}^{\prime}$ is not omissive and has $\ell$ as the starter (respectively, reactor), $C_{j}$ is starter-omission-recurrent (respectively, reactor-omission-recurrent) for $\mathcal{S}(\mathcal{P})$.

Proof. First we will insert at most $k$ omissive interactions into $I$ (thus building an infinite sequence that satisfies property (1)) in such a way as to satisfy property (3). Then we will choose $t$ so as to satisfy property (2).

We will construct $I^{\prime}$ incrementally by an inductive procedure. Suppose we have constructed $I^{\prime}$ up to $i_{j}^{\prime}$, and let $C_{j}$ be the configuration of $\ell$ and $a$ after executing the first $j$ interactions of $I^{\prime}$ starting from $C_{0}$ (the base case is with $j=0)$. Suppose also that the partial sequence $\left(i_{1}^{\prime}, \ldots, i_{j}^{\prime}\right)$ has been obtained by adding some omissive interactions to some initial sub-sequence of $I$, say $\left(i_{1}, \ldots, i_{v(j)}\right)$ (with $v(0)=0$, i.e., in the base case the subsequence is empty). Let $i_{v(j)+1}$ have $\ell$ as its starter (respectively, reactor). Then, if $C_{j}$ is starter-omission-recurrent (respectively, reactor-omission-recurrent), we set $i_{j+1}^{\prime}=i_{v(j)+1}$ and $v(j+1)=v(j)+1$. Otherwise, we let $i_{j+1}^{\prime}$ be omissive on both sides with $\ell$ as the starter (respectively, reactor), and we set $v(j+1)=v(j)$.

We claim that, if we continue this process indefinitely, we put at most $k$ omissive interactions in $I^{\prime}$. Indeed, suppose that an omissive interaction $i_{j}^{\prime}$ with $\ell$ as the starter (respectively, reactor) has been inserted in $I^{\prime}$, which means that $C_{j-1}$ is not a starter-omission-recurrent (respectively, reactor-omission-recurrent) configuration. By 
definition of omission-recurrent configuration, $\ell$ will never get the simulated state it had in $C_{j-1}$ after executing $i_{j}^{\prime}$. It follows that the same state will contribute to the addition of at most one omissive interaction to $I^{\prime}$. Since the possible states for $\ell$ are at most $k$, there can be at most $k$ omissive interactions in $I^{\prime}$.

We now have to decide when to stop the incremental construction of $I^{\prime}$. Recall that the execution of $I$ starting from $C_{0}$ is globally fair, and observe that adding finitely many omissive interactions to it preserves its global fairness. So, by definition of simulator, the simulated states of $\ell$ and $a$ also change in a globally fair way as they execute $I^{\prime}$. Therefore, at some point, they will conclude a simulated interaction, changing their local states according to $\delta_{\mathcal{P}}$. At this point we stop the construction of $I^{\prime}$, obtaining a sequence of finite length $t$ that satisfies all three properties.

Theorem 1. A system of agents, each of which has a finite amount of memory, cannot simulate every two-way protocol in the $\mathrm{T}_{3}$ model (hence in all the omissive models), even with the presence of a leader and under the $\diamond \mathrm{NO}$ adversary.

Proof. We will show that the Pairing Protocol $\mathcal{P}_{I P}$ cannot be simulated in $\mathrm{T}_{3}$. Suppose by contradiction that there is a simulator $\mathcal{S}\left(\mathcal{P}_{I P}\right)$ for it, and let us consider a system of two agents $\ell$ and $a$, where $\ell$ is the leader. Let $C_{0}$ be an initial configuration in which $\ell$ has simulated state $p$ and $a$ has simulated state $c$, and let $I$ be an omission-less infinite sequence of interactions for the two agents whose execution starting from $C_{0}$ is globally fair. According to $\mathcal{P}_{I P}$, eventually $I$ will make both $\ell$ and $a$ change simulated state to $\perp$ and $c s$, respectively.

Let us apply Lemma 1 to $\mathcal{S}\left(\mathcal{P}_{I P}\right), C_{0}$, and $I$, which yields a sequence of interactions $I^{\prime}=\left(i_{1}^{\prime}, \ldots, i_{t}^{\prime}\right)$ of length $t$, some of which are omissive on both sides. The sequence $I^{\prime}$ guarantees that both agents will change simulated state to $c s$ and $\perp$, as per property (2). Let $C_{j}$ be the configuration of the two agents after executing the first $j$ interactions of $I$ starting from configuration $C_{0}$.

Now we construct a larger system of agents: $\left\{\ell, a, b_{1}, b_{2}, \ldots, b_{m}, d\right\}$, where $m=2^{t}$. Let $C_{0}^{\prime}$ be the configuration of this system in which $\ell$ and $a$ have the same state as in $C_{0}$ and all other agents have the same state as $a$. We will show how to modify $I^{\prime}$ by inserting some extra interactions in it, obtaining an expanded sequence $I^{\prime \prime}$ that involves also the other members of the system (as opposed to only $\ell$ and $a$ ). We will then show that executing $I^{\prime \prime}$ makes the simulator behave in a way that is not compatible with the Pairing Problem.

We will construct $I^{\prime \prime}$ inductively by inserting a sequence of interactions before each interaction $i_{j}^{\prime} \in I^{\prime}$. Say we have already done so up to $i_{j}^{\prime}$, with $0 \leq j<t$ (the base case being with $j=0$ ). Executing $I^{\prime \prime}$ up to this point from configuration $C_{0}^{\prime}$ makes the system reach configuration $C_{j}^{\prime}$. Let $w(j)=m / 2^{j}$, and suppose that agents $a, b_{1}, b_{2}$, $\ldots, b_{w(j)}$ have the same state in $C_{j}^{\prime}$ (this is certainly true for $j=0$ ). Also suppose that, in $C_{j}^{\prime}, \ell$ and $a$ have the same state as in $C_{j}$ (again, this is true for $j=0$ ). As we construct $I^{\prime \prime}$ and $j$ increases, we will also prove that these properties are preserved.

Now consider the next interaction $i_{j+1}^{\prime}$, which could be either non-omissive or omissive on both sides. Next we are going to explain what interactions we add to $I^{\prime \prime}$ between $i_{j}^{\prime}$ and $i_{j+1}^{\prime}$.

Suppose that $i_{j+1}^{\prime}$ is omissive on both sides, and let $\ell$ be the starter and $a$ the reactor. Then we introduce in $I^{\prime \prime}$ (right after $i_{j}^{\prime}$ ) the sequence of interactions $\left(d, b_{1}\right),\left(d, b_{2}\right), \ldots,\left(d, b_{w(j) / 2}\right)$, omissive on both sides. Finally we introduce $i_{j+1}^{\prime}$ into $I^{\prime \prime}$. If $a$ is the starter and $\ell$ the reactor, we insert the same interactions, but with starter and reactor exchanged. It is immediate to see that, after executing these interactions from configuration $C_{j}^{\prime}$, agents $a, b_{1}, b_{2}, \ldots, b_{w(j+1)}$ have equal states. Indeed, they have the same state in $C_{J}^{\prime}$, and then they all execute one omissive interaction as starters or as reactors. Moreover, in $C_{j+1}^{\prime}, \ell$ and $a$ have the same state as in $C_{j+1}$.

Suppose that $i_{j+1}^{\prime}$ is not omissive, and let $\ell$ be the starter and $a$ the reactor. By property (3) of $I^{\prime}$, configuration $C_{j}$ is starter-omission-recurrent. Let $q_{\ell}$ be the state of $\ell$ in $C_{j}$ (and therefore in $C_{j}^{\prime}$ ). By definition of starteromission-recurrent configuration, there exists a sequence of interactions $I^{*}=\left(i_{1}^{*}, i_{2}^{*}, \ldots, i_{z}^{*}\right)$, with $i_{1}^{*}$ omissive on both sides and having $\ell$ as the starter and $a$ as the reactor, such that the state of $\ell$ becomes $q_{\ell}$ again if $I^{*}$ is executed from configuration $C_{j}$ (and hence from $C_{j}^{\prime}$ ). Note that the same happens if the partner of $\ell$ in the interactions of $I^{*}$ is not $a$ but any of the $b_{x}$ 's, with $1 \leq x \leq w(j)$, since all these agents have the same state in $C_{j}^{\prime}$ by inductive hypothesis. Given these premises, we introduce in $I^{\prime \prime}$ (right after $i_{j}^{\prime}$ ) the following interactions.

- For all $1 \leq x \leq w(j) / 2$, we insert:

- the interaction $\left(\ell, b_{x}\right)$, omissive on $\ell$ 's side;

- the interaction $\left(d, b_{x+w(j) / 2}\right)$, omissive on both sides;

- the sequence of interactions $\left(i_{2}^{*}, i_{3}^{*}, \ldots, i_{z}^{*}\right)$, with $b_{x+w(j) / 2}$ as $\ell$ 's partner instead of $a$. 
- Finally, we insert $i_{j+1}^{\prime}$.

The case in which $\ell$ is the reactor of $i_{j+1}^{\prime}$ and $a$ the starter is handled in a similar fashion, but we exchange starter and reactor in the interactions that we add to $I^{\prime \prime}$. Suppose now that the system executes the above sequence of interactions starting from configuration $C_{j}^{\prime}$, and let us focus on the sub-system consisting of $\ell$ and $b_{x+w(j) / 2}$. The agent $\ell$ starts in state $q_{\ell}$ and then executes an omissive interaction, while $b_{x+w(j) / 2}$ executes another omissive interaction. Together, these two interactions have the same effect on $\ell$ and $b_{x+w(j) / 2}$ as $i_{1}^{*}$ (note that the partners of $\ell$ and $b_{x+w(j) / 2}$ were irrelevant, because the interactions were omissive on their sides). Then $\ell$ and $b_{x+w(j) / 2}$ execute all the interactions of $I^{*}$ except $i_{1}^{*}$. Since $b_{x+w(j) / 2}$ started in the same state as $a$, by definition of $I^{*}$ it follows that the state of $\ell$ is reset again to $q_{\ell}$ after this sequence. By induction, this is true for all $1 \leq x \leq w(j) / 2$. Finally, the state of $\ell$ correctly changes according to $i_{j+1}^{\prime}$ as it interacts with $a$. On the other hand, $a$ and all the $b_{x}$ 's see $\ell$ exactly once when it is in state $q_{\ell}$ and, since they have the same state in $C_{j}^{\prime}$, they also have the same state in $C_{j+1}^{\prime}$.

We have shown that agents $a, b_{1}, b_{2}, \ldots, b_{w(j)}$ have the same state in $C_{j}^{\prime}$ for all $1 \leq j \leq t$. In particular, for $j=t$, we have that $w(j)=m / 2^{t}=1$, which means that $a$ and $b_{1}$ have the same state in $C_{t}^{\prime}$. In turn, the simulated state of $a$ in $C_{t}^{\prime}$ is the same as in $C_{t}$, i.e., cs. Since at the beginning there was only one agent with simulated state $p$ (i.e., $\ell$ ), and now we have two agents with simulated state $c s$, we have violated the safety property of the Pairing Problem, meaning that $\mathcal{S}\left(\mathcal{P}_{I P}\right)$ cannot be a simulator.

As there is only a finite number of omissions in $I^{\prime \prime}$, this sequence of interactions can be extended to an infinite one with the addition of non-omissive interactions, which is compatible with the $\diamond \mathbf{N O}$ adversary.

\subsection{Impossibility with Infinite Memory}

For this case we can show that simulation is impossible in the omissive two-way model without detection, and thus in 10 .

Theorem 2. A system of agents, each of which has an infinite amount of memory, cannot simulate every two-way protocol in the $\mathrm{T}_{1}$ model (hence in $\mathrm{IO}$ ), even with the presence of a leader and under the $\diamond \mathrm{NO}$ adversary.

Proof. We will show that the Pairing Protocol $\mathcal{P}_{I P}$ cannot be simulated in $\mathrm{T}_{1}$. Let $\mathcal{S}\left(\mathcal{P}_{I P}\right), \ell, a, C_{0}$, and $I=$ $\left(i_{1}, i_{2}, \ldots\right)$ be defined as in the first paragraph of the proof of Theorem 1. By definition of simulator and by the Pairing Problem, if we execute $I$ from $C_{0}$ according to $\mathcal{S}\left(\mathcal{P}_{I P}\right)$, at some point we reach a configuration in which $\ell$ has simulated state $\perp$ and $a$ has simulated state $c s$. Say that this happens after executing $i_{j}$, and let $I_{j}=\left(i_{1}, i_{2}, \ldots, i_{j}\right)$.

Let us now extend the system with a third agent $b$, initially having the same state as $a$ in $C_{0}$, and let us show how to insert interactions into $I_{j}$ involving $b$ as well, in order to obtain a contradictory finite sequence of interactions $I^{\prime}$. Recall that $I$ is omission-less, and consider the interaction $i_{x}$, with $1 \leq x \leq j$. If $i_{x}=(\ell, a)$, we insert the interaction $(\ell, b)$ right before it, with omission on $\ell$ 's side. If $i_{x}=(a, \ell)$, we insert the interaction $(b, \ell)$ right before it, again with omission on $\ell$ 's side.

Since omissions are undetectable, it is easy to see that the extended sequence $I^{\prime}$ will make $\ell$ undergo the same state transitions as $I_{j}$ (but at half the "speed"). On the other hand, $a$ and $b$ will always see $\ell$ in the same state and will never see each other, so they will both have the same state throughout the execution of $I^{\prime}$. It follows that $a$ and $b$ will eventually have simulated state $c s$, which violates the safety property of the Pairing Problem.

Note that the sequence $I^{\prime}$ contains finitely many omissions, and therefore it can be extended to an infinite sequence that is compatible with the $\diamond \mathbf{N O}$ adversary.

Observation 1. Since in 10 there are no omissions, the statement of Theorem 2 for the 10 model trivially extends to the scenario in which the number of omissions in the sequence of interactions is known in advance by the agents.

\section{Simulation in Omissive Models}

In this section we are going to make use of a result that appears in 24] as Theorem 4.5. This theorem assumes each agent to have a unique ID, which is a non-negative integer, as part of its local state.

Theorem 3. Assuming IO, unique IDs, and $\mathcal{O}(\log (\max I D))$ bits of memory on each agent (where max ID is the maximum ID in the system), there exists a simulator for every two-way protocol, even under the $\mathbf{U O}$ adversary. 
What this theorem says is that, if the agents initially have unique IDs, they can perform a simulation of any two-way protocol, even if the simulation runs in the weakest model, IO, and against the strongest adversary, UO.

In this section we assume the presence of a leader and we show that, in certain models, we can implement a naming algorithm, i.e., an algorithm that assign unique IDs to all agents. Once an ID has been assigned to an agent, it cannot change. Therefore, the naming algorithm and the simulator of Theorem 3 can be combined into a single protocol and can even run in parallel: if an agent has no ID yet, the simulator simply ignores every interaction involving this agent. By global fairness, eventually all agents will have unique IDs, and the simulation will finally involve the entire system, producing a globally fair simulated execution.

The protocols will be presented using an algorithmic style: for each interaction of the form $\left(a_{s}, a_{r}\right)$, the starter agent $a_{s}$ executes function Upon Event Starter sends() and the reactor agent $a_{r}$ executes Upon Event Reactor delivers $\left(\operatorname{var}^{s}\right)$, where $v a r^{s}$ is the variable var in the local state of agent $a_{s}$.

\subsection{Naming Algorithm with Infinite Memory}

If the leader has infinite memory, it can implement a simple naming algorithm under certain models. Since Theorem 2 already states the impossibility of simulation under models $\mathrm{T}_{1}$ and $\mathrm{IO}$, we will assume model $\mathrm{I}_{1}$ or model $\mathrm{I}_{2}$. Constructing a simulator for these models will imply the existence of a simulator for all other models except $T_{1}$ and IO (refer to Figure 2).

Theorem 4. Assuming $\mathrm{I}_{1}$ or $\mathrm{I}_{2}$, the presence of a leader, and an infinite amount of memory on each agent, there exists a simulator for every two-way protocol, even under the UO adversary.

Proof. By the above discussion, it suffices to implement a naming algorithm. Each agent has a local variable $m y_{-} I D$ and the leader also has a second variable next_ID. The leader has $m y_{-} I D=0$ and next_ID $=1$, while all other agents initially have $m y_{-} I D=\perp$. Every time the leader detects the proximity of another agent (i.e., it applies function $g$ to its own state), it increments the variable next_ID. Every time an agent sees the state of the leader (i.e., it applies function $f$ ), it sets its own $m y_{-} I D$ to the value found in the leader's next_ID variable.

Since the leader increments next_ID every time it is involved in an interaction (even if the interaction is omissive on the other side), no two agents can get the same ID. Moreover, by global fairness, all agents will eventually see the leader in a non-omissive interaction, and will therefore acquire an ID.

\subsection{Naming Algorithms with Knowledge on Omissions}

Now we assume that agents have only a finite amount of memory, but they know in advance a finite upper bound $L$ on the number of omission faults that the adversary is going to insert in interactions that involve the leader. Note that the adversary can still be UO even if only finitely many omissive interactions involve the leader.

\subsubsection{Naming Algorithm for $I_{1}$ and $I_{2}$}

We refine the naming algorithm of Theorem 4 to cope with the fact that now memory is bounded by a function of $L$ and the size of the system, $n$. It is worth mentioning that the precise value of $n$ is not known to the agents, and $L$ is only an upper bound on the number of omissions involving the leader, not necessarily the exact number.

Theorem 5. Assuming $\mathrm{I}_{1}$ or $\mathrm{I}_{2}$, the presence of a leader, knowledge of an upper bound $L$ on the number of omission failures in interactions that involve the leader, and $\Theta(L \log n L)$ bits of memory on each agent (where $n$ is the number of agents), there exists a simulator for every two-way protocol, even under the UO adversary.

Proof. We implement the naming algorithm presented in Figure 3 . Compared to the algorithm of Theorem 4 , here the leader has an array of $L+1$ next_I $D$ variables, as opposed to only one. This array is initialized to $[1,2, \ldots, L+1]$ and, when an ID is assigned, the corresponding entry of the array will be incremented by $L+1$, so that no two equal IDs are ever be generated.

All entries of next_ID are initially unlocked: this information is stored in the leader's Boolean array locked. The active ID is defined as the unlocked entry of next_ID having minimum index, if there is any (line 10 ). This is the ID that will tentatively be assigned next. Whenever the leader detects the proximity of another agent (i.e., it executes function $g$ on its own state, or function Upon Event Starter sends in the algorithm of Figure 3), it locks the active entry of next_ID (line 12). The purpose of locking an entry of next_ID (as opposed to just incrementing it as in 
Theorem (4) is that the leader cannot allow its value to grow indefinitely, because now memory is limited. Instead, the leader will make the entry temporarily inactive, and will keep it on hold until it gathers more information in the following interactions.

On the other hand, if an agent $a$ sees the leader (i.e., it executes function $f$ or function Upon Event Reactor delivers in Figure 3), and $a$ does not have an ID yet, then it assigns itself the active ID from the leader's next_ID variable (line 30). So, the next time the leader sees $a$, it will read its new ID and it will know that the corresponding entry of next_ID can be unlocked (line 23) and its value can be incremented by $L+1$ (line 24).

It may happen that the leader is involved in an omissive interaction, and therefore the entry of next_ID that it locks will never be unlocked again. However, this can happen at most $L$ times, while the array has $L+1$ entries.

This is not sufficient yet, because the same agent $a$ may see the leader multiple times in a row and cause all entries of next_ID to become locked. If $a$ only stores one ID, it will have no way to tell the leader that more than one entry of next_ID has to be unlocked. This is why $a$ also has a variable called redundant, which is a Boolean array that will store information on all the active entries of next_ID that $a$ sees after receiving an ID. So, if the agent $a$ already has an ID and it sees the leader again, it sets to true the entry of reduntant corresponding to the active ID of the leader (line 32 .

Now, suppose that the leader sees that $a$ has an entry of redundant set to true. This implies that the corresponding entry of next_ID is currently locked and should be unlocked. However, this cannot be done right away: the leader wants to give $a$ an "acknowledgment", so that $a$ will set the entry of redundant to false first. This is to prevent the scenario in which the entry of next_ID gets unlocked, becomes active, another agent $b$ sees it, and takes it as its own ID. If then the leader sees a again (still with redundant on true), it will unlock the entry of $n e x t_{-} I D$. Then perhaps yet another agent $c$ will see the leader, getting the same ID as $b$.

To prevent such an incorrect behavior, the leader has another variable array called waiting, in which it stores the IDs of the agents that should reset their redundant variables. So, when the leader sees that $a$ has some entry of redundant set to true, it stores the (unique) ID of $a$ in the corresponding entry of waiting (line 18). Then, when $a$ sees the leader again and reads its own ID in the waiting array, it knows that it has to set to false the corresponding entries of redundant (line 34). Finally, when the leader sees a again and notices that the entry of redundant has been set to false, it can reset the corresponding entry of waiting (line 20) and unlock the entry of new_ID (line 21).

The fact that the algorithm does not give the same ID to two different agents follows from the observation that at most one agent can keep an entry of new_ID locked at any given time, which in turn follows from the way the two variables redundant and waiting function together. If no omission occurs and the leader is observed by some agent $a$, then $a$ will store information about the currently active ID. If $a$ takes this ID for itself, that entry of next_ID will be incremented before any other agent can get the same ID. If $a$ has already an ID, the entry of next_ID will remain locked until $a$ has reset its own redundant variable. Moreover, the fact that the algorithm will eventually assign every agent an ID immediately follows from the global fairness of the adversarial scheduler.

Since the IDs in the next_ID array increase by $L+1$ every time one is assigned, and since there are $n$ agents in total, the value of every ID is $\mathcal{O}(n L)$. Hence, $\mathcal{O}(L \log n L)$ bits of memory are required to store each agent's arrays, and $\mathcal{O}(\log n L)$ more bits are required to run the simulator of Theorem 3 . The total amount of memory needed per agent is therefore $\mathcal{O}(L \log n L)$ bits.

\subsubsection{Naming Algorithm for $\mathrm{T}_{1}$}

Observe that the previous naming algorithm does not work for model $\mathrm{T}_{1}$, and Theorem 2 does not hold when some kind of upper bound on omissions is known.

Theorem 6. Assuming $\mathrm{T}_{1}$, the presence of a leader, knowledge of an upper bound $L$ on the number of omission failures in interactions that involve the leader, and $\Theta(L \log n L)$ bits of memory on each agent (where $n$ is the number of agents), there exists a simulator for every two-way protocol, even under the UO adversary.

Proof. It is sufficient to give a naming algorithm. We modify the one used in Theorem 4 to work in $\mathrm{T}_{1}$ with $\mathcal{O}(L \log n L)$ memory. The leader has the same two local variables, but each other agent has an array my_ID of $L+1$ local variables, each of which is initially set to $\perp$. If an agent $a$ sees the leader, and the local array $m y_{-} I D$ of $a$ has some entries still set to $\perp$, then $a$ changes one of them from $\perp$ to the value of the leader's next_ID variable. On the other hand, if the leader sees an agent whose local array $m y_{-} I D$ has some entries still set to $\perp$, it increments $n e x t \_I D$. When all entries of an agent's array $m y \_I D$ have been set, the entire array is taken as the agent's ID. 


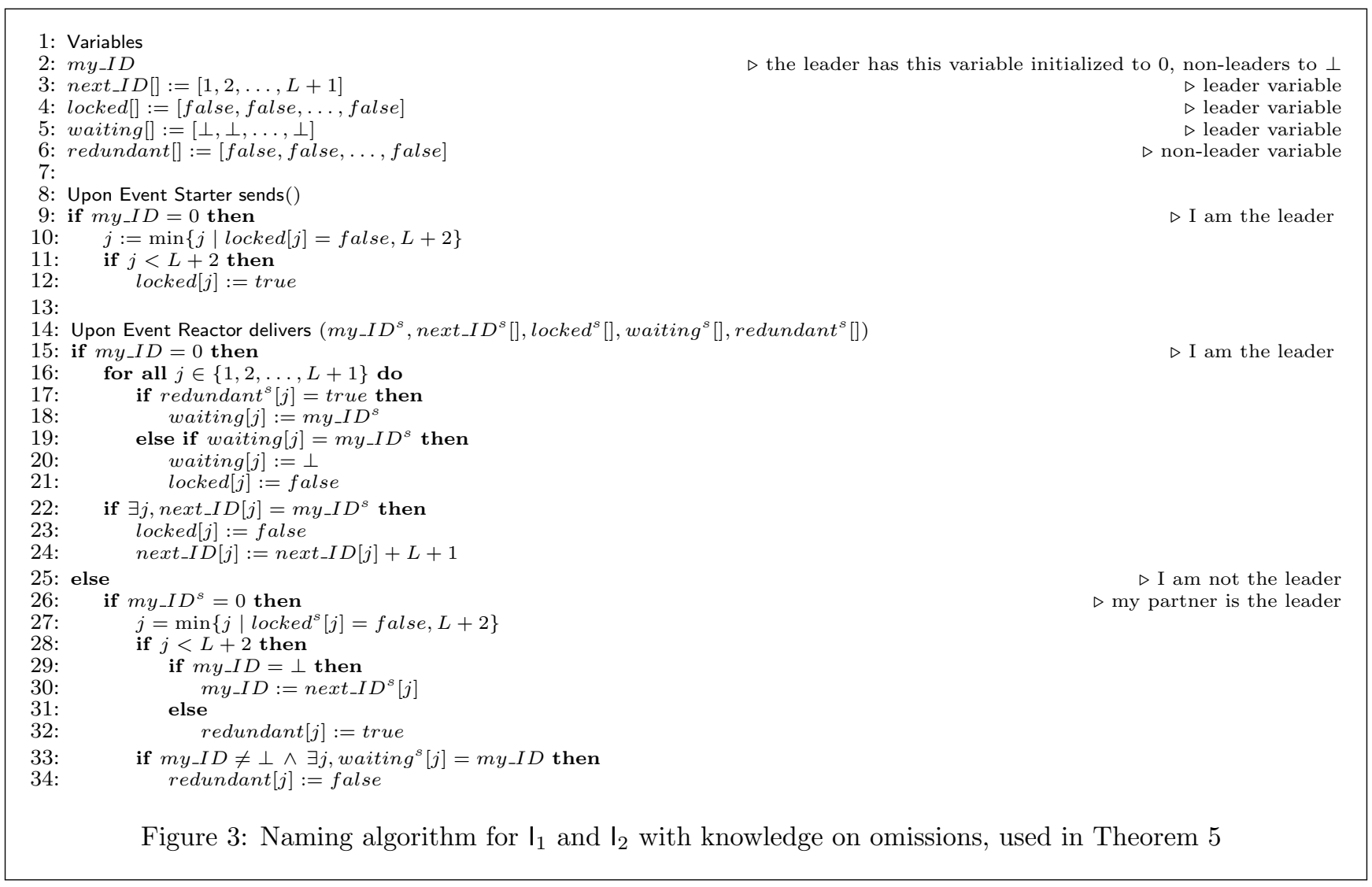

1: Variables

2: role

3: state $_{\mathcal{P}}:=$ initial_state $_{\mathcal{P}}$

4: token $:=\perp$

6: Upon Event Starter sends()

7: token $:=\perp$

8: if role $=$ leader then

9: $\quad$ role := available

10: else if role $=$ moving then

11: role $:=$ available

2: else if role $=$ starter then

13: role $:=$ pending

14:

15: Upon Event Reactor delivers (role ${ }^{s}$, state $_{\mathcal{P}}^{s}$, token $^{s}$ )

16: token $:=$ token $^{\text {s }}$

17: if role $^{\mathrm{s}}=$ leader then

18: $\quad$ role $:=$ moving

9: else if role $^{s}=$ moving then

role $:=$ starter

else if role $^{s}=$ starter then

token $:=$ state $P$

state $_{\mathcal{P}}:=f_{r}\left(\right.$ state $_{\mathcal{P}}^{s}$, state $\left._{\mathcal{P}}\right)$

else if role $=$ pending $\wedge$ token $\neq \perp$ then

state $_{\mathcal{P}}:=f_{s}\left(\right.$ state $_{\mathcal{P}}$, token $)$

role $:=$ leader

token $:=\perp$ $\triangleright$ the leader has this variable initialized to leader, non-leaders to available

Figure 4: Simulation protocol for IT with finite memory, used in Theorem 7 
Since the execution is globally fair, eventually all agents will have their $m y_{-} I D$ array completely set. Observe that, whenever the leader increments next_ID, there is an agent $a$ that removes one occurrence of $\perp$ from its local array $m y_{-} I D$, unless the interaction is omissive on $a$ 's side. But there can only be $L$ such omissive interactions, which means that the maximum value of next_ID will be $\mathcal{O}(n L)$. So, $\Theta(L \log n L)$ bits of memory are enough for an agent to store its local array my_ID. By Theorem 3 , combining this naming algorithm with the simulator does not require more than $\Theta(L \log n L)$ bits of memory on each agent.

Suppose for a contradiction that two agents receive equal IDs. Therefore, both agents have observed the leader $L+1$ times, and at the $j$ th observation both agents must have read the same value in variable next_ID, for all $1 \leq j \leq L+1$. So, the leader has failed to increment next_ID for at least $L+1$ times, implying that there have been $L+1$ omissive interactions involving the leader, which contradicts the theorem's assumptions. Thus all agents receive distinct IDs, and the naming algorithm is correct.

\section{Simulation for IT}

Notice that IT is the only finite-memory model for which the impossibility result of Theorem 1 does not hold (see Figure 22. It turns out that in this model we can implement a simulator that sequentializes the simulated two-way interactions via a token-passing technique.

Theorem 7. Assuming IT, the presence of a leader, and a constant amount of memory on each agent, there exists a simulator for every two-way protocol, even under the UO adversary.

Proof. The simulation algorithm is reported in Figure 4. Suppose we are given a two-way protocol $\mathcal{P}$ whose transition function is $\delta_{\mathcal{P}}\left(a_{s}, a_{r}\right)=\left(f_{s}\left(a_{s}, a_{r}\right), f_{r}\left(a_{s}, a_{r}\right)\right)$. In our simulator, each agent has a local variable called state $_{\mathcal{P}}$, which is the state of $\mathcal{P}$ that the agent is simulating, plus an auxiliary variable role, which is used to coordinate the simulation. Initially, the role of one agent is leader, while all others are available. When the leader meets another agent $a$, the leadership is "transferred" to $a$ : the role of the leader becomes available (line 9), and the role of $a$ becomes moving (line 18). Note that the leader does not have to see the state of $a$ to perform this operation.

The next agent $b$ that sees $a$ becomes the starter of a new simulated interaction: the role of $b$ becomes starter (line 20) and the role of $a$ becomes available again (line 11). Now, the first agent $c$ that observes $b$ becomes the reactor of the simulated interaction: it executes function $f_{r}$ on its own simulated state using $b$ 's simulated state as part of the input (line 23), while $b$ 's role becomes pending (line 13).

Now, in order for $b$ to perform its side of the simulated transition, it has to retrieve the simulated state that $c$ had before transitioning. To deliver this information to $b$, the agent $c$ stores its own simulated state in a variable called token before performing the transition (line 22). Now, as soon as an agent sees $c$, it copies the token (line 16), while $c$ erases its own copy (line 7). This token circulation protocol is executed until the token reaches $b$.

When $b$ finally obtains the token, it uses it as part of the input to function $f_{s}$ and changes its simulated state accordingly (line 25). Now both sides of the simulated transition have been performed correctly, and $b$ resets its role to leader (line 26) and destroys the token (line 27). At this point we have exactly one agent whose role is leader, while all other agents have role available, as we had at the beginning. The next steps of the simulation are thus performed in the same fashion.

Note that, if the two-way protocol $\mathcal{P}$ has a constant number $k$ of states, then our simulator has $\mathcal{O}\left(k^{2}\right)$ states, independently of the size of the system.

The correctness of the simulator can be proven by observing that, due to the uniqueness of the leader, there is at most one pending transition at all times. Moreover, any agent in the system can become the starter (including the leader itself), thanks to the extra step that creates an agent with role moving: any non-leader agent can become moving, and then any agent (including the original leader) can become starter. Note that this is not true if the system consists of $n=2$ agents (in this case the leader will necessarily become the starter of every simulated interaction), but recall that the definition of simulator assumes that $n>2$ (cf. Section 2.3).

We have to prove that the system will perform infinitely many simulated interactions, in such a way that the simulated execution is globally fair. By the global fairness of the simulator, a token will certainly be created and will be passed around by the agents; again by global fairness, the token will eventually reach the agent with role $=$ starter, and the simulated interaction will be concluded. The global fairness of the entire simulated execution also follows immediately from the global fairness of the simulator itself. 


\section{References}

[1] D. Alistarh and R. Gelashvili. "Polylogarithmic-time leader election in population protocols", 42nd International Colloquium on Automata, Languages and Programming, ICALP, 2015, pp. 479-491.

[2] D. Alistarh, R. Gelashvili, and M. Vojnovic. "Fast and exact majority in population protocols", 34th Annual ACM Symposium on Principles of Distributed Computing, PODC, 2015, pp. 47-56.

[3] D. Angluin, J. Aspnes, Z. Diamadi, M. J. Fischer, and R. Peralta. "Computation in networks of passively mobile finite-state sensors", Distributed Computing, vol. 18(4), 2006, pp. 235-253.

[4] D. Angluin, J. Aspnes, and D. Eisenstat. "On the power of anonymous one-way communication", 9th International Conference on Principles of Distributed Systems, OPODIS, 2005, pp. 396-411.

[5] D. Angluin, J. Aspnes, and D. Eisenstat. "Stably computable predicates are semilinear", 25th Annual ACM Symposium on Principles of Distributed Computing, PODC, 2006, pp. 292-299.

[6] D. Angluin, J. Aspnes, and D. Eisenstat. "A simple population protocol for fast robust approximate majority", Distributed Computing, vol. 21(2), 2008, pp. 87-102.

[7] D. Angluin, J. Aspnes, and D. Eisenstat. "Fast computation by population protocols with a leader", Distributed Computing, vol. 21(3), 2008, pp. 61-75.

[8] J. Aspnes and E. Ruppert. "An introduction to population protocols", Bulletin of the European Association for Theoretical Computer Science, vol. 93, 2007, pp. 98-117.

[9] J. Beauquier, J. Burman, S. Clavière, and D. Sohier. "Space-optimal counting in population protocols", 29th International Symposium on Distributed Computing, DISC, 2015, pp. 631-649.

[10] J. Beauquier, J. Burman, and S. Kutten. "A self-stabilizing transformer for population protocols with covering", Theoretical Computer Science, vol. 412(33), 2011, pp. 4247-4259.

[11] O. Bournez, P. Chassaing, J. Cohen, L. Gerin, and X. Koegler. "On the convergence of population protocols when population goes to infinity", Applied Mathematics and Computation, vol. 215(4), 2009, pp. 1340-1350.

[12] S. Cai, T. Izumi, and K. Wada. "How to prove impossibility under global fairness: on space complexity of self-stabilizing leader election on a population protocol model", Theory of Computing Systems, vol. 50(3), 2012, pp. 433-445.

[13] A. Casteigts, P. Flocchini, W. Quattrociocchi, and N. Santoro. Time-varying graphs and dynamic networks. International Journal of Parallel, Emergent and Distributed Systems, vol. 27(5), 2012, pp. 387-408.

[14] I. Chatzigiannakis, S. Dolev, S. P. Fekete, O. Michail, and P. G. Spirakis. "Not all fair probabilistic schedulers are equivalent", 13th International Conference on Principles of Distributed Systems, OPODIS, 2009, pp. 33-47.

[15] I. Chatzigiannakis, O. Michail, S. Nikolaou, and A. Pavlogiannis. "Passively mobile communicating machines that use restricted space", Theoretical Computer Science, vol. 412(46), 2011, pp. 6469-6483.

[16] I. Chatzigiannakis, O. Michail, S. Nikolaou, A. Pavlogiannis, and P. G. Spirakis. "All symmetric predicates in NSPACE $\left(n^{2}\right)$ are stably computable by the mediated population protocol model", 35th International Symposium on Mathematical Foundations of Computer Science, MFCS, 2010, pp. 270-281.

[17] I. Chatzigiannakis, O. Michail, and P. G. Spirakis. "Stably decidable graph languages by mediated population protocols", 12th International Symposium on Stabilization, Safety, and Security of Distributed Systems, SSS, 2010, pp. 252-266.

[18] I. Chatzigiannakis, O. Michail, and P. G. Spirakis. "Mediated population protocols", Theoretical Computer Science, vol. 412(22), 2011, pp. 2434-2450.

[19] I. Chatzigiannakis, O. Michail, and P. G. Spirakis. "New models for population protocols", Synthesis Lectures on Distributed Computing Theory, Morgan \& Claypool, 2011. 
[20] I. Chatzigiannakis and P. G. Spirakis. "The dynamics of probabilistic population protocols", 22nd International symposium on Distributed Computing, DISC, 2008, pp. 498-499.

[21] H.-L. Chen, R. Cummings, D. Doty, and D. Soloveichik. "Speed faults in computation by chemical reaction networks", 28th International Symposium on Distributed Computing, DISC, 2014, pp. 16-30.

[22] C. Delporte-Gallet, H. Fauconnier, and R. Guerraoui. "What dependability for networks of mobile sensors?" 1st Workshop on Hot Topics in System Dependability, HotDep, 2005, p. 8.

[23] C. Delporte-Gallet, H. Fauconnier, R. Guerraoui, and E. Ruppert. "When birds die: making population protocols fault-tolerant", 2nd IEEE International Conference on Distributed Computing in Sensor Systems, DCOSS, 2006, pp. 51-66.

[24] G. A. Di Luna, P. Flocchini, T. Izumi, T. Izumi, N. Santoro, and G. Viglietta. "On the power of weaker pairwise interaction: fault-tolerant simulation of population protocols", arXiv:1610.09435 [cs.DC], 2016.

[25] M. Fischer and H. Jiang. "Self-stabilizing leader election in networks of finite-state anonymous agents", 10th International Conference on Principles of Distributed Systems, OPODIS, 2006, pp. 395-409.

[26] R. Guerraoui and E. Ruppert. "Even small birds are unique: population protocols with identifiers", Technical Report CSE-2007-04, York University, 2007.

[27] R. Guerraoui and E. Ruppert. "Names trump malice: tiny mobile agents can tolerate byzantine failures", 36th International Colloquium on Automata, Languages and Programming, ICALP, vol. 16(2), 2009, pp. 484-495. 